\title{
Gravitational instabilities of uniform black strings in AdS space
}

\author{
Aditya Dhumuntarao $\odot^{*}$ and Rafid Mahbub $\odot^{\dagger}$ \\ School of Physics and Astronomy, University of Minnesota, Minneapolis, Minnesota 55455, USA
}

(Received 27 October 2021; accepted 2 January 2022; published 3 February 2022)

Locally $\mathrm{AdS}_{d-1} \times \mathbb{R}$ uniform black strings (UBS) in the presence of a massless scalar field are believed to avoid the onset of the Gregory-Laflamme (GL) instability in $d \geq 4$ as no tachyonic modes exist in the spectrum of the Laplace-Beltrami operator. We present analytic and numerical evidence of GL modes in the Lichnerowicz spectrum indicating that $\mathrm{AdS}_{d-1}$ UBSs are classically and thermodynamically unstable at the linear level in $d>4$. In $d=4$, we confirm that uniform $\mathrm{BTZ}_{3}$ strings are indeed stable as previously suggested. This supports that linear instabilities of black strings are triggered only if tachyonic modes exist in the Lichnerowicz spectrum. At the end state of the instability, $\operatorname{AdS}_{d-1}$ UBSs of finite length may tunnel to a $\mathrm{SAdS}_{d}$ black hole or converge onto a novel nonuniform $\mathrm{AdS}_{d}$ black string. We conjecture that weak cosmic censorship is violated if the nonuniform solution is an exact $\mathrm{AdS}_{d}$ black funnel and compute entropy estimates in $d>4$ as evidence.

DOI: 10.1103/PhysRevD.105.L041501

\section{INTRODUCTION}

Black objects with translationally invariant horizons suffer from classical instabilities. A famous example is the Gregory-Laflamme (GL) instability of Ricci-flat black strings in $d>4$, with horizon topologies $S^{d-3} \times S^{1}$, where tachyonic modes develop in the Lichnerowicz operator spectrum $\left(\Delta_{L}\right)$ under generic long wavelength perturbations [1-4]. These classical instabilities persist to four spacetime dimensions and higher [5-21] for black strings with asymptotics that are conformal to $\mathrm{AdS}_{d-1} \times \mathbb{R}$. There is strong numerical evidence that GL modes lead to the horizon developing a finite time pinch-off-constituting a violation of weak cosmic censorship [22]. For black strings with nontrivial matter configurations, tachyonic modes are also observed in the Laplace-Beltrami operator $(\square)$ (see [18-21]). It is generally believed that the spectra of scalar operators on black string backgrounds are correlated. Thus, finding tachyonic modes in the matter sector is a necessary and sufficient condition for classical instability and indeed, several numerical and analytic situations exist where such instabilities were sourced from $\operatorname{Spec}(\square)$ only [19-21].

These linear instabilities are correlated to local thermodynamic instabilities. In its modern form, correlated stability states, for black strings with mass $M$ and conserved

\footnotetext{
dhumu002@umn.edu †mahbu004@umn.edu
}

Published by the American Physical Society under the terms of the Creative Commons Attribution 4.0 International license. Further distribution of this work must maintain attribution to the author(s) and the published article's title, journal citation, and DOI. Funded by SCOAP . charges $Q_{A}$, classical instabilities are triggered precisely when a positive eigenvalue exists in the Hessian of the microcanonical entropy $S\left(M, Q_{A}\right)$ [19,20]. For unique black strings with conserved charges, correlated stability has demonstrated that tachyonic modes develop in the spectrum of scalar operators precisely when the heat capacity becomes negative [6-9,13,23-27] and enjoys a general proof for asymptotically flat spacetimes [28].

Recently, a new class of locally $\operatorname{AdS}_{d-1} \times \mathbb{R}$ uniform black string $\left(\mathrm{AdS}_{d-1} \mathrm{UBS}\right)$ solutions in the presence of a massless scalar field has furnished two interesting questions $[29,30]$. First, no such tachyonic modes were identified in $\operatorname{Spec}(\square)$ leading to the conjecture that these solutions are linearly stable in four spacetime dimensions and higher [30]. This result is clearly in tension with earlier studies, which have numerically and analytically confirmed a tachyonic mode in $\Delta_{L}$ for black strings that are locally conformal to $\mathrm{AdS}_{d-1} \times \mathbb{R}$ [3-18]. As the stability analysis performed in $[29,30]$ primarily focused on $\operatorname{Spec}(\square)$, it is natural to ask does a GL mode persist in $\operatorname{Spec}\left(\Delta_{L}\right)$ in four spacetime dimensions and higher?

Secondly, the $\operatorname{AdS}_{d-1}$ UBS is thermodynamically unstable when the radius of the string is sufficiently thin but remains classically stable in this regime. It is well known that nonunique black brane configurations with exotic scalar charge are counterexamples to correlated stability [12]. However, the $\operatorname{AdS}_{d-1}$ UBS is a unique configuration without a conserved charge associated with the massless scalar $[29,30]$, naturally raising the question are these solutions a new counterexample to the stronger form of correlated stability?

In this paper, we present an analytic and numerical study of the classical and thermodynamic instability of locally 
$\operatorname{AdS}_{d-1} \times \mathbb{R}$ uniform black strings coupled to a massless scalar. In concordance with the study initiated in [30], we confirm that no GL modes exist in the $\operatorname{Spec}(\square)$. However, we find evidence of tachyonic modes in $\operatorname{Spec}\left(\Delta_{L}\right)$ in $d>4$, suggesting that the $\operatorname{AdS}_{d-1} \times \mathbb{R}$ UBS is classically unstable. We also find a positive eigenvalue in $\operatorname{Hess}(S)$ which, together with the GL modes in $\Delta_{L}$, is sufficient to rescue correlated stability in $d>4$. The reason the scalar operator spectra are uncorrelated may be understood by performing Kaluza-Klein (KK) compactifications along the flat direction. The resulting tower of masses for the scalar fluctuations satisfy the Breitenlöhner-Freedman bound on the locally $\operatorname{AdS}_{d-1}$ background [31,32]. Hence, deformations from the matter sector do not grow sufficiently fast to trigger an instability, whereas the same is not true for propagating tensor modes in $\operatorname{Spec}\left(\Delta_{L}\right)$. To our knowledge, this is the first example where the spectra of scalar operators are uncorrelated for AdS black strings in the presence of nontrivial matter. This suggests tachyonic modes in $\operatorname{Spec}(\square)$ are sufficient, but not necessary as previously thought, to generate classical instabilities, and it supports earlier studies that linear black string instabilities are triggered only if tachyonic modes exist in $\operatorname{Spec}\left(\Delta_{L}\right)$.

\section{THE MODEL}

We parameterize the $\mathrm{AdS}_{d-1}$ UBS solution by

$$
\begin{aligned}
\mathcal{S} & =\frac{1}{16 \pi G_{d}} \int\left[R-2 \Lambda_{\text {bulk }}-\frac{1}{2}(\partial \sigma)^{2}\right] \sqrt{-g} \mathrm{~d}^{d} x, \\
\mathrm{~d} s^{2} & =\frac{(d-2)}{(d-1)} \frac{L_{d}^{2}}{\ell_{d-1}^{2}}\left[\mathrm{~d} z^{2}-V(r) \mathrm{d} t^{2}+\frac{\mathrm{d} r^{2}}{V(r)}+r^{2} \mathrm{~d} s_{S^{d-3}}^{2}\right], \\
V(r) & =1-m r^{4-d}+r^{2} \ell_{d-1}^{-2}, \quad \sigma(z)=\sqrt{2(d-2)}\left(z / \ell_{d-1}\right) .
\end{aligned}
$$

The bulk and boundary AdS length scales are set via $\Lambda_{\text {bulk }}=-\frac{(d-1)(d-2)}{2 L_{d}^{2}}$ and $\Lambda_{\text {bdy }}=-\frac{(d-2)(d-3)}{2 \ell_{d-1}^{2}}$. We consider a finite string with $z \in \mathbb{I}=\left[-\pi L_{z} / 2, \pi L_{z} / 2\right]$ and restrict our attention to $d \geq 4$. The UBS is a solution to the equations,

$$
R_{a b}=\frac{2 \Lambda_{\text {bulk }}}{d-2} g_{a b}+\frac{1}{2} \partial_{a} \sigma \partial_{b} \sigma \quad \square \sigma=0,
$$

provided the massless scalar $\sigma$ satisfies Neumann boundary conditions. Clearly, the scalar modifies $\Lambda_{\text {bulk }}$ along the $z$ direction and additional flat directions $x_{i}$ may be appended at the expense of adding scalars $\sigma_{i}$ with identical asymptotics. Henceforth, the volume of the space transverse to the UBS horizon is unity, $\left(16 \pi G_{d}\right)^{-1} \int_{S^{d-3} \times \mathbb{I}} \sqrt{\gamma}=1$ [33].

\section{CORRELATED STABILITY}

On constant $z$ hypersurfaces, the geometry describes an $\mathrm{SAdS}_{d-1} \mathrm{BH}$ where the largest positive root of $V\left(r_{+}\right)=0$ designates the horizon. The mass and entropy may then be directly computed using standard formulas on the codimension one surface,

$M=(d-3) r_{+}^{d-4}\left[E_{S^{d-3}}+\frac{r_{+}^{2}}{\ell_{d-1}^{2}}\right], \quad S=4 \pi r_{+}^{d-3}$,

where $E_{S^{d-3}}$ is the energy of the sphere, which vanishes in $d=4$ and is unity otherwise. As noted in [30], there is no conserved charge associated with the scalar. Hence, the thermodynamic potential is simply $S \equiv S(M)$ in the microcanonical ensemble, and searching for positive eigenvalues in $\operatorname{Hess}(S)$ is equivalent to identifying a regime where $S(M)$ is convex. However, for AdS black objects, there is no simple expression for the entropy $[19,20]$. Rather, assuming strictly positive temperatures (which is reasonable for $\mathrm{SAdS}_{d-1}$ black strings), the stability requirement may be rephrased in terms of finding negative eigenvalues in the Hessian of $M(S)$, which is simply the statement that the heat capacity becomes negative. We find in $d=4$, $M(S)=\left(S / 4 \pi \ell_{d-1}\right)^{2}$ and in $d>4$,

$$
M(S)=(d-3)\left(\frac{S}{4 \pi}\right)^{\frac{d-4}{d-3}}\left[1+\frac{(S / 4 \pi)^{\frac{2}{d-3}}}{\ell_{d-1}^{2}}\right] .
$$

A routine computation of the heat capacity $C=T\left(\partial_{S}^{2} M\right)^{-1}$ where $T=V^{\prime}\left(r_{+}\right) / 4 \pi$, shows that $M(S)$ is concave in $d>4$ for $r_{+}<r_{+}^{c}=\sqrt{(d-4) /(d-2)} \ell_{d-1}$. Hence, sufficiently thin UBSs with $S\left(r_{+}\right)<S\left(r_{+}^{c}\right)=4 \pi r_{+}^{c}$ are thermodynamically unstable in $d>4$. In $d=4$, the solution is a uniform $\mathrm{BTZ}_{3}$ string with a mass gap $M \geq 0$ separating the black string from the $\operatorname{AdS}_{3}(M=-1)$ background, which has a strictly positive heat capacity [34,35]. Via correlated stability, this naively suggests tachyonic modes condense in $\operatorname{Spec}\left(\square, \Delta_{L}\right)$ for horizons $r_{+}<r_{+}^{c}$ in $d>4$, whereas the $\mathrm{BTZ}_{3}$ UBS is stable regardless of the horizon size. Hence, the GL instability should have a lower critical dimension of $d=5$. Some evidence of this is observed for $\mathrm{BTZ}_{3}$ black strings, which are locally conformal to $\mathrm{AdS}_{3} \times \mathbb{R}$ [7-9]. In $4 \geq d$, Einstein - (A) $\mathrm{dS}_{d-1}$ gravity is topological. Thus, metric perturbations will not contain propagating degrees of freedom and should reduce to a pure gauge transformation. We study these expectations analytically and numerically shortly.

\section{LINEAR CLASSICAL STABILITY}

We show that tachyonic modes in $\operatorname{Spec}\left(\square, \Delta_{L}\right)$ are uncorrelated on the $\operatorname{AdS}_{d-1}$ UBS background, and the $\mathrm{BTZ}_{3} \mathrm{UBS}$ is stable. We consider perturbing the metric and scalar field around the background Eq. (1),

$$
g_{a b}\left(x^{a}\right)=\bar{g}_{a b}\left(x^{a}\right)+\epsilon H_{a b}\left(x^{a}\right)+\mathcal{O}\left(\epsilon^{2}\right),
$$




$$
\sigma\left(x^{a}\right)=\bar{\sigma}(z)+\epsilon \Phi\left(x^{a}\right)+\mathcal{O}\left(\epsilon^{2}\right),
$$

where $\left(\bar{g}_{a b}, \bar{\sigma}\right)$ satisfies the equations of motion (2). To $\mathcal{O}(\epsilon)$, the perturbations on the $\operatorname{AdS}_{d-1}$ UBS background satisfy

$$
\begin{gathered}
\square \Phi \equiv-\frac{1}{V} \frac{\partial^{2} \Phi}{\partial t^{2}}+\frac{1}{r^{d-3}} \frac{\partial}{\partial r}\left(r^{d-3} V \frac{\partial \Phi}{\partial r}\right)+\frac{\partial^{2} \Phi}{\partial z^{2}}=0, \\
\Delta_{L} H_{a b}=-\frac{4 \Lambda}{d-2} H_{a b}+2 \partial_{(a} \bar{\sigma} \partial_{b)} \Phi,
\end{gathered}
$$

where the Lichnerowicz operator in the transverse gauge is

$$
\Delta_{L} H_{a b} \equiv \square H_{a b}+2 \bar{R}_{a c b d} H^{c d}-2 \bar{R}_{(a}^{c} H_{b) c}^{c} .
$$

Now, identifying tachyonic modes in $\operatorname{Spec}\left(\square, \Delta_{L}\right)$ is tantamount to finding regular solutions $\left(H_{a b}, \Phi\right)$, which have a growing mode instability [1-5]. As the UBS solution is spherically symmetric and has a Killing isometry along the flat direction, we search for growing modes in the $s$-wave gauge with the expansion,

$$
\begin{gathered}
\Phi\left(x^{a}\right)=e^{i m_{n} z+\Omega t} \phi(r), \\
H_{a b}\left(x^{a}\right)=e^{i m_{n} z+\Omega t}\left(\begin{array}{cccc}
h_{t t} & h_{t r} & h_{t z} & \mathbf{0} \\
h_{t r} & h_{r r} & h_{r z} & \mathbf{0} \\
h_{t z} & h_{r z} & h_{z z} & \mathbf{0} \\
\mathbf{0} & \mathbf{0} & \mathbf{0} & \boldsymbol{h}_{i j}
\end{array}\right) .
\end{gathered}
$$

Here, $m_{n}=\left(n / L_{z}\right), \boldsymbol{h}_{i j} \mathrm{~d} \theta^{i} \mathrm{~d} \theta^{j} \equiv K(r) \mathrm{d} s_{S^{d-3}}^{2}$, and $h_{\mu \nu} \equiv$ $h_{\mu \nu}(r)$ along the directions transverse to $S^{d-3}$. In the presence of matter, we are allowed to work in the transverse gauge $H_{; b}^{a b}=\frac{1}{2} H^{; a}$, where $H=\bar{g}^{a b} H_{a b}$. Lastly, in order for the initial value problem to be well posed, the domain of dependence will include $\mathscr{I}^{+}$on the conformal boundary of the $\mathrm{SAdS}_{d-1} \mathrm{BH}$ when prescribing data $[1,2,5]$.

\section{MATTER PERTURBATIONS}

It is straightforward to show that no tachyonic modes may be found in $\operatorname{Spec}(\square)$ obeying regularity conditions near horizon $r \approx r_{+}$and asymptotically $r \rightarrow \infty$ [30]. Evaluating Eq. (7) on the ansatz Eq. (11), one finds

$$
V \phi^{\prime \prime}+\left[\frac{d-3}{r} V+V^{\prime}\right] \phi^{\prime}-\left[m_{n}^{2}+\frac{\omega^{2}}{V}\right] \phi=0 .
$$

Regularity conditions enforce the behavior $\phi \sim\left(r-r_{+}\right)^{\alpha}$ near horizon and $\phi \sim r^{-\beta}$ asymptotically with $\alpha, \beta \in \mathbb{R}^{+}$ [36]. As a result, the only way to achieve this behavior is if $\phi(r)$ possesses a maximum at some radial position $r_{\star}>r_{+}$. At the turning point, $\phi^{\prime}\left(r_{\star}\right)=0$, and hence, we have

$$
V\left(r_{\star}\right) \phi^{\prime \prime}\left(r_{\star}\right)=\left[m_{n}^{2}+\frac{\omega^{2}}{V\left(r_{\star}\right)}\right] \phi\left(r_{\star}\right) .
$$

However, for this to be a maximum, $V\left(r_{\star}\right) \phi^{\prime \prime}\left(r_{\star}\right)<0$, but no maximum may exist as $V\left(r_{\star}\right)>0$ for $r_{\star}>r_{+}$.

We generalize the stability argument given in [30] by performing a KK compactification of the flat direction. One would then find a tower of masses $\left(\square^{\left.\text {SAdS }_{d-1}-m_{n}^{2}\right) \Phi=0}\right.$ from which it is direct to see the Breitenlöhner-Freedman stability condition $m_{n}^{2}=\left(n / L_{z}\right)^{2}>-\frac{(d-2)^{2}}{4 \ell_{d-1}^{2}}$ is satisfied $[31,32]$. Hence, the $\operatorname{AdS}_{d-1}$ UBS solution is indeed stable to scalar perturbations in $d \geq 4$ independent of the coordinate gauge. As such, we eliminate this perturbation in our search for an instability [2].

\section{METRIC PERTURBATIONS}

The metric perturbations are quite involved. Nonetheless, it is possible to present the salient arguments, detailed in the Supplemental Material [37], which identify the growing mode instability. As the scalar perturbation decouples, the metric perturbations become trace free $\bar{g}^{a b} H_{a b}=0$. Then gauge conditions, in conjunction with eliminating stable perturbations, can be used to reduce Eq. (8) to a single ODE,

$$
A_{m_{n}, \Omega}(r) h_{t r}^{\prime \prime}(r)+B_{m_{n}, \Omega}(r) h_{t r}^{\prime}(r)+C_{m_{n}, \Omega}(r) h_{t r}(r)=0 .
$$

The conditions on the radial boundary and the horizon are sensitive to the dimension and on $\Lambda_{\text {bdy }}$. The qualitative difference between $d=4$ and $d>4$ is best understood in the limit $\left(\Lambda_{\text {bulk }}, \Lambda_{\text {bdy }}\right) \rightarrow 0$, where one is supposed to recover the instability of Schwarzschild $d_{-1}$ uniform black strings [38]. In $d=4$, no Schwarzschild ${ }_{3}$ UBS exists in the spectrum of Einstein gravity, where as the same is not true in $d>4$. Next, the dependence of the regularity condition on $\Lambda_{\text {bdy }}$ may be inferred in terms of the control parameter $\mu_{+}=r_{+} / \ell_{d-1}$. For $\mu_{+} \gg 1$, the radius of the $\operatorname{AdS}_{d-1}$ UBS is large and requires sharply growing regular perturbations to destabilize the solution, whereas $\mu_{+} \ll 1$ corresponds to thin UBS solutions, which are easier to perturb. We perform the parameter search in the latter regime in order to make contact with correlated stability. The conditions on the horizon and radial boundary may be found by solving Eq. (14) on the various asymptotics. We find

$$
\begin{array}{r}
r \rightarrow r_{+}: \begin{cases}\mathcal{A}_{ \pm}\left(m_{n}\right)\left(r-r_{+}\right)^{-1 \pm \frac{r_{+} \Omega}{d-4} \sqrt{1-\varepsilon}}, & d>4 \\
\mathcal{C}_{ \pm}\left(m_{n}\right)\left(r-r_{+}\right)^{-1 \pm \frac{r_{+} \Omega}{2 \mu_{+}^{2}}}, & d=4,\end{cases} \\
r \rightarrow \infty: \begin{cases}\mathcal{B}_{ \pm}\left(m_{n}\right) e^{ \pm m_{n}^{2} r / \sqrt{m_{n}^{2}-\Omega^{2}}}, & d>4 \\
\mathcal{D}_{ \pm}\left(m_{n}\right) e^{ \pm \mathrm{i} m_{n}^{2} r / \sqrt{\mu_{+}^{2} m_{n}^{2}+\Omega^{2}}}, & d=4,\end{cases}
\end{array}
$$



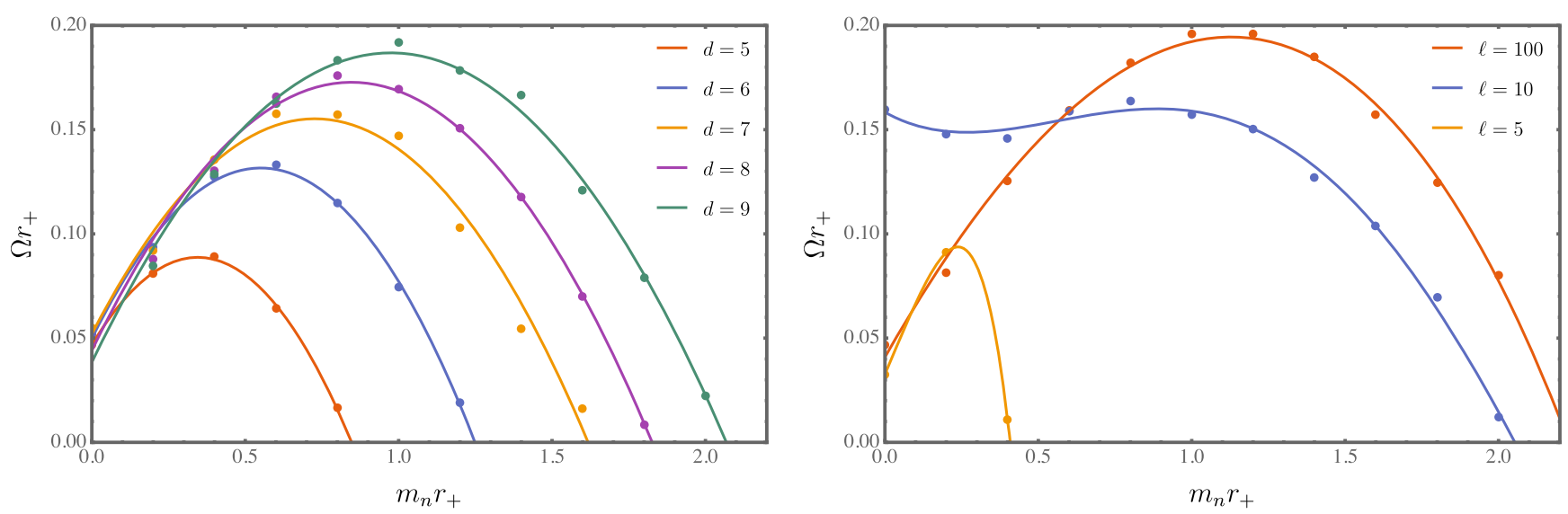

FIG. 1. Left: Plot of $\left(m_{n}, \Omega\right)$ with $r_{+}=2.0$ and $\ell=50$ for which the ratio $\mathcal{R}$ changes sign in $9 \leq d \leq 5$. The points correspond to values calculated numerically indicating an instability. The lines are up-to-quadratic polynomial fits to illustrate the trend. Right: The instability in $d=10$ for various values of $\ell$. As $\mu_{+} \sim \mu_{+}^{c}$, the instability is terminated.

where $\varepsilon=\frac{(d-2)}{(d-4)} \mu_{+}^{2}$. In $d>4$, the near horizon condition suggests a real regular solution exists provided $\varepsilon<1$ and $\Omega<(d-4) / r_{+} \sqrt{1-\varepsilon}$. The condition on $\varepsilon$ is analytic evidence of correlated stability as the control parameter is bounded above precisely by the thermodynamic instability $\mu_{+}^{2}<\frac{(d-4)}{(d-2)} \equiv\left(r_{+}^{c} / \ell_{d-1}\right)^{2}$. Near horizon, one recovers the familiar Schwarzschild ${ }_{d-1}$ UBS behavior when $\mu_{+}=0$ $[1,2]$. In the far horizon, the solution differs due to the conformal structure on the asymptotically $\operatorname{AdS}_{d-1}$ surface.

In $d=4$, it is evident the perturbation is pure gauge- the asymptotic solutions are oscillatory rather than smoothly damped. Near horizon, instabilities are exponentially damped out from the spectrum as regular solutions require $\Omega<\frac{2 \mu_{+}^{2}}{r_{+}} \ll 1$. We believe this behavior to be related to the lack of propagating gravitational degrees of freedom in lower dimensions. It is natural to understand this as analytic evidence suggesting the classical stability of $\mathrm{BTZ}_{3}$ UBSs, which agrees with the expectations provided from correlated stability.

The goal is now to perform a parameter search on $\left(m_{n}, \Omega\right)$, which yields regular solutions, i.e., tachyonic modes in $\operatorname{Spec}\left(\Delta_{L}\right)$. We numerically integrate Eq. (14) (see the Supplemental Material [37]) to compute the instability pairs $\left(m_{n}, \Omega\right)$ using an adaptive Runge-Kutta-Fehlberg (RK45) routine in Mathematica. A backward integration is carried out between $r_{1}=200.0$ and $r_{2}=r_{+}+10^{-5}$ with the seed solution set by the decaying branch $h_{t r} \sim e^{-m_{n}^{2} r / \sqrt{m_{n}^{2}-\Omega^{2}}}$. The existence of an instability is detected with a sign change in the ratio $\mathcal{R}=\mathcal{A}_{-} / \mathcal{A}_{+}$ $[1,2]$, where

$\mathcal{R}=\frac{\left(\frac{r_{+} \Omega}{d-4} \sqrt{1-\varepsilon}-1\right) h_{t r}-\left(r-r_{+}\right) h_{t r}^{\prime}}{\left(\frac{r_{+} \Omega}{d-4} \sqrt{1-\varepsilon}-1\right) h_{t r}+\left(r-r_{+}\right) h_{t r}^{\prime}}\left(r-r_{+}\right)^{\frac{2 r_{+} \Omega}{d-4} \sqrt{1-\varepsilon}}$.
We confirm the $\mathrm{AdS}_{d-1}$ UBS solution is indeed unstable in $10 \geq d \geq 5$, and in $d=10$, we studied the threshold of this instability for various control parameters. We found no such instability in $d=4$ or beyond $r_{+}^{c} / \ell_{d-1}$; the results are summarized in Fig. [1].

\section{COMMENTS ON WEAK COSMIC CENSORSHIP VIOLATION}

Naturally, one may ask which geometry arises at the end state of this instability, an Schwarzschild - $\mathrm{AdS}_{d}$ black hole $\left(\mathrm{SAdS}_{d} \mathrm{BH}\right)$ or a novel nonuniform $\mathrm{AdS}_{d}$ black string $\left(\mathrm{AdS}_{d}\right.$ nUBS) configuration, and if a violation of weak cosmic censorship occurs during the transition, especially in $d=4$. As it turns out, the existence of a GL mode is insufficient to provide a violation of weak cosmic censorship [39] (see [14,15] where GL points exist, but a tunneling event is not expected to occur). One is further required to compute the entropy of the nUBS solution and determine its dominance in the phase diagram. It is then reasonable to conjecture that weak cosmic censorship is violated if $(i)$ a GL mode exists and (ii) the nonuniform solution has a subdominant entropy relative to the uniform solution $\left(S_{\mathrm{nUBS}}<S_{\mathrm{UBS}}\right)$.

In the absence of a dynamical scalar, a suggestive set of nonuniform saddles can be constructed by foliating $\mathrm{AdS}_{d}$ in terms of $\mathrm{AdS}_{d-1}$ slices and then replacing each slice with $\mathrm{SAdS}_{d-1}$ black holes. The resulting solutions to Eq. (1) are exact $\mathrm{AdS}_{d}$ black funnels (BF) [16,17],

$\begin{aligned} \mathrm{d} s_{\mathrm{BF}}^{2} & =\frac{\left(L_{d} / \ell_{d-1}\right)^{2}}{\cos \left(z / \ell_{d-1}\right)^{2}}\left[\mathrm{~d} z^{2}-V(\hat{r}) \mathrm{d} \hat{t}^{2}+\frac{\mathrm{d} \hat{r}^{2}}{V(\hat{r})}+\hat{r}^{2} \mathrm{~d} s_{S^{d-3}}^{2}\right], \\ V(\hat{r}) & =1-\hat{m} \hat{r}^{4-d}-\hat{r}^{2} \ell_{d-1}^{2}, \quad \sigma(z)=\sigma_{0},\end{aligned}$

which are locally conformal to $\operatorname{AdS}_{d-1} \times \mathbb{R}$. The phase diagram now contains at least [14] three black objects with distinct horizon topologies $\mathcal{H}$ and scalar minima: 

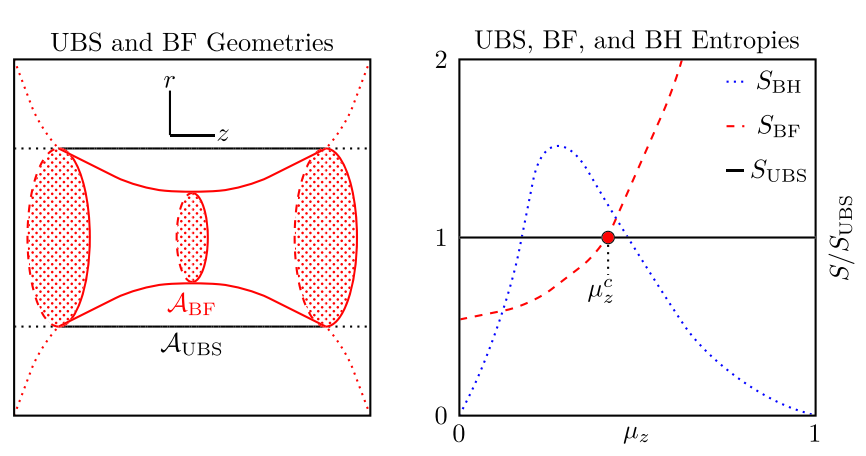

FIG. 2. Left: Diagram of coincident UBS and BF geometries within the strip $z \in\left[-\pi L_{z} / 2, \pi L_{z} / 2\right]$ at equal mass. Right: The UBS is entropically dominant over the BF solution for $\mu_{z}<\mu_{z}^{c}$. The $\mathrm{BH}$ entropy is observed to follow the above trend.

$\operatorname{SAdS}_{d}$ BH with $\left(\sigma=\sigma_{0}, \mathcal{H}=S^{d-2}\right), \mathrm{AdS}_{d}$ BF with $\left(\sigma=\sigma_{0}, \mathcal{H}=\Omega_{z}^{2}\left(S^{d-3} \times \mathbb{R}\right)\right)$, and $\operatorname{AdS}_{d-1}$ UBS with $\left(\sigma \propto z, \mathcal{H}=S^{d-3} \times \mathbb{R}\right)$. On thermodynamic grounds, transitions between these geometries is expected at a critical point $M_{\star}$ [14]. Clearly, a violation of weak cosmic censorship is expected in any first order transition between black string and black hole phases, whereas fragmentation does not occur during transitions between black string phases that share conformally equivalent horizon topologies. If the BF phase is entropically dominant, then an energy barrier will separate the UBS and BH phases. Hence, the statement of weak cosmic censorship violation amounts to finding regimes where $S_{\mathrm{BF}}<S_{\mathrm{UBS}}$ so that transitions between black strings and black holes occur. Such a regime will depend on the control parameter $\mu_{z}=L_{z} / \ell_{d-1}$. One should expect that area difference of the UBS and BF strips, with length $z \in\left[-\pi L_{z} / 2, \pi L_{z} / 2\right]$, should be negative for sufficiently small $\mu_{z}$ (see Fig. 2). We confirm this via entropy estimates in $d \geq 5$. The thermodynamics read

$$
\begin{gathered}
M_{\mathrm{BF}}=(d-3) \hat{r}_{+}^{d-4}\left[E_{S^{d-3}}+\frac{\hat{r}_{+}^{2}}{\ell_{d-1}^{2}}\right] I_{d}\left(\mu_{z}\right), \\
S_{\mathrm{BF}}=4 \pi \hat{r}_{+}^{d-3} I_{d}\left(\mu_{z}\right),
\end{gathered}
$$

where $\quad I_{d}\left(\mu_{z}\right)=(2 \pi)^{-1}\left(\frac{d-1}{d-2}\right)^{(d-2) / 2} \int_{-\pi / 2}^{\pi / 2} \cos \left(\mu_{z} x\right)^{2-d} \mathrm{~d} x$, which is convergent for $\mu_{z}<1$ [see Eq. (22)]. Along the line $M_{\mathrm{BF}}=M_{\mathrm{UBS}}$, the entropy difference may be estimated in the stable regime $\left(\mu_{+}, \hat{\mu}_{+}\right) \gg E_{S^{d-3}}$ via

$$
\Delta S\left(\mu_{z}\right)=S_{\mathrm{UBS}}-S_{\mathrm{BF}} \approx S_{\mathrm{UBS}}\left(1-I_{d}\left(\mu_{z}\right)^{\frac{1}{d-2}}\right) .
$$

The UBS and BF phases exchange dominance when $I_{d}\left(\mu_{z}^{c}\right)=1$. Under these conditions, it is reasonable to conjecture that weak cosmic censorship is violated provided the nonuniform saddle corresponds to an exact $A d S_{d}$ black funnel geometry.

\section{CONCLUSIONS}

We find the spectra of scalar operators on locally $\mathrm{AdS}_{d-1} \times \mathbb{R}$ uniform black strings supported by a massless scalar $[29,30]$ are indeed uncorrelated in $d \geq 5$, contrary to prior expectations [18-21], and this solution falls into the class of black strings covered by correlated stability in $d \geq 4$. Tachyonic modes in the Laplace-Beltrami spectrum appear to be only sufficient to generate classical instabilities. This suggests that black string instabilities are triggered only if there exist tachyonic modes in $\operatorname{Spec}\left(\Delta_{L}\right)$. Lastly, we have found a regime of intermediate $\mathrm{AdS}_{d-1}$ UBS solutions, which are entropically favorable over exact $\mathrm{AdS}_{d} \mathrm{BF}$ and compete directly with Schwarzschild - $\operatorname{AdS}_{d}$ BHs. Although a complete numerical study of the Einstein - Dilaton - $\mathrm{AdS}_{d}$ model is needed to elucidate if weak cosmic censorship is violated, the thermodynamic argument presents some preliminary evidence that the instability can tunnel a UBS to a BH. In $\mathrm{AdS}_{d}$ with a constant scalar, it is well known that additional black objects, such as black droplets, exist in the thermodynamic phase space [14]. These black objects do not change the qualitative argument given here as the horizons in such cases are partitioned, and hence, it is reasonable to conjecture a violation of weak cosmic censorship in $d \geq 5$. It is of interest to understand which configuration the $\operatorname{AdS}_{d-1}$ UBS will ultimately converge onto.

\section{ACKNOWLEDGMENTS}

The authors acknowledge the use of the diffgeo Mathematica package for their symbolic tensor calculations [40]. We thank Jorge Santos, Robie Hennigar, Robert Mann, and Matthew Headrick for discussions and hospitality. We are supported by the University of Minnesota Doctoral Dissertation Fellowship. A. D. is supported by the National Science Foundation Graduate Research Fellowship under Grant No. 00039202.

Note Added.-After the completion of the present analysis, we were made aware of similar work carried out independently in $d=5$ [41]. The analysis of the $\mathrm{AdS}_{4}$ UBS solution there involved a power series expansion of the metric perturbation $h_{t r}$ in order to search for an instability. We would like to thank the authors of [30] for sharing their preprint along with their helpful insights on the subject. 
[1] R. Gregory and R. Laflamme, Black Strings and p-Branes are Unstable, Phys. Rev. Lett. 70, 2837 (1993).

[2] R. Gregory and R. Laflamme, The instability of charged black strings and p-branes, Nucl. Phys. B428, 399 (1994).

[3] G. Gibbons and S.A. Hartnoll, Gravitational instability in higher dimensions, Phys. Rev. D 66, 064024 (2002).

[4] H. Kudoh, Origin of black string instability, Phys. Rev. D 73, 104034 (2006).

[5] R. Gregory, Black string instabilities in anti-de sitter space, Classical Quantum Gravity 17, L125 (2000).

[6] L. Liu and B. Wang, Stability of BTZ black strings, Phys. Rev. D 78, 064001 (2008).

[7] G. Kang, On the stability of black strings / branes, in 11th Workshop on General Relativity and Gravitation (2002), https://inspirehep.net/literature/583243.

[8] T. Hirayama and G. Kang, Stable black strings in anti-de sitter space, Phys. Rev. D 64, 064010 (2001).

[9] G. Kang and J. Lee, Classical stability of black D3 branes, J. High Energy Phys. 03 (2004) 039.

[10] R. B. Mann, E. Radu, and C. Stelea, Black string solutions with negative cosmological constant, J. High Energy Phys. 09 (2006) 073.

[11] Y. Brihaye, T. Delsate, and E. Radu, On the stability of AdS black strings, Phys. Lett. B 662, 264 (2008).

[12] J. J. Friess, S. S. Gubser, and I. Mitra, Counterexamples to the correlated stability conjecture, Phys. Rev. D 72, 104019 (2005).

[13] A. Bernamonti, M. M. Caldarelli, D. Klemm, R. Olea, C. Sieg, and E. Zorzan, Black strings in $\operatorname{AdS}(5)$, J. High Energy Phys. 01 (2008) 061.

[14] D. Marolf and J. E. Santos, Phases of holographic Hawking radiation on spatially compact spacetimes, J. High Energy Phys. 10 (2019) 250.

[15] Y. Bea, O. J. C. Dias, T. Giannakopoulos, D. Mateos, M. Sanchez-Garitaonandia, J. E. Santos, and M. Zilhao, Crossing a large- $N$ phase transition at finite volume, J. High Energy Phys. 02 (2021) 061.

[16] V. E. Hubeny, D. Marolf, and M. Rangamani, Hawking radiation from AdS black holes, Classical Quantum Gravity 27, 095018 (2010).

[17] V. E. Hubeny, D. Marolf, and M. Rangamani, Black funnels and droplets from the AdS C-metrics, Classical Quantum Gravity 27, 025001 (2010).

[18] H. S. Reall, Classical and thermodynamic stability of black branes, Phys. Rev. D 64, 044005 (2001).

[19] S. S. Gubser and I. Mitra, The evolution of unstable black holes in anti-de sitter space, J. High Energy Phys. 08 (2001) 018 .

[20] S. S. Gubser and I. Mitra, Instability of charged black holes in Anti-de Sitter space, arXiv:hep-th/0009126.

[21] S. F. Ross and T. Wiseman, Smeared D0 charge and the Gubser-Mitra conjecture, Classical Quantum Gravity 22, 2933 (2005).
[22] L. Lehner and F. Pretorius, Black Holes in Higher Dimensions, edited by G. T. Horowitz (Cambridge University Press, 2012), 10.1017/CBO9781139004176.

[23] S. S. Gubser and A. Ozakin, Universality classes for horizon instabilities, J. High Energy Phys. 05 (2003) 010.

[24] T. Hirayama, G. Kang, and Y. Lee, Classical stability of charged black branes and the gubser-mitra conjecture, Phys. Rev. D 67, 024007 (2003).

[25] A. Buchel, A holographic perspective on Gubser-Mitra conjecture, Nucl. Phys. B731, 109 (2005).

[26] U. Miyamoto, Analytic evidence for the Gubser-Mitra conjecture, Phys. Lett. B 659, 380 (2008).

[27] S. Chen, K. Schleich, and D. M. Witt, Thermodynamics and stability of flat anti-de sitter black strings, Phys. Rev. D 78, 126001 (2008).

[28] S. Hollands and R. M. Wald, Stability of black holes and black branes, Commun. Math. Phys. 321, 629 (2013).

[29] A. Cisterna and J. Oliva, Exact black strings and p-branes in general relativity, Classical Quantum Gravity 35, 035012 (2018).

[30] A. Cisterna, C. Henríquez-Báez, and J. Oliva, Stabilizing homogeneous black strings in AdS, J. High Energy Phys. 01 (2020) 052.

[31] P. Breitenlohner and D. Z. Freedman, Stability in gauged extended supergravity, Ann. Phys. (N.Y.) 144, 249 (1982).

[32] P. Breitenlohner and D. Z. Freedman, Positive energy in anti-De Sitter backgrounds and gauged extended supergravity, Phys. Lett. 115B, 197 (1982).

[33] This yields the normalization

$$
G_{d}=\frac{\omega_{d-3} L_{z}}{8}\left(\frac{d-2}{d-1}\right)^{\frac{1}{2}(d-2)}\left(\frac{L_{d}}{\ell_{d-1}}\right)^{d-2}
$$

which is convenient to use when computing the thermodynamics.

[34] M. Banados, C. Teitelboim, and J. Zanelli, The Black Hole in Three-Dimensional Space-Time, Phys. Rev. Lett. 69, 1849 (1992).

[35] A. M. Frassino, R. B. Mann, and J. R. Mureika, Lowerdimensional black hole chemistry, Phys. Rev. D 92, 124069 (2015).

[36] P. Pani, Advanced methods in black-hole perturbation theory, Int. J. Mod. Phys. A 28, 1340018 (2013).

[37] See Supplemental Material at http://link.aps.org/ supplemental/10.1103/PhysRevD.105.L041501 for an analysis of the metric perturbations.

[38] We thank Matthew Headrick for bringing this to our attention.

[39] We would like to thank Jorge Santos for valuable communications on this matter.

[40] M. Headrick, Diffgeo Mathematica package.

[41] C. Henríquez-Báez, On the stability of homogeneous black strings in AdS, arXiv:2110.08328. 